\title{
A REMARK ON EXTREMALLY $\mu$-DISCONNECTED GENERALIZED TOPOLOGICAL SPACES
}

\author{
BRIJ KISHORE TYAGI AND HARSH V. S. CHAUHAN
}

\begin{abstract}
A more general definition of extremally $\mu$-disconnected generalized topological space [3] is introduced and its properties are studied. We have further improved the definitions of generalized open sets [1] and upper(lower) semi-continuous functions defined for a generalized topological space in [5]. In this generalized framework we obtain the analogues of results in [1,3,5]. Examples of extremally $\mu$-disconnected generalized topological spaces are given.
\end{abstract}

\section{INTRODUCTION}

Extremally disconnected topological spaces defined by Stone [6] turned out to be non-trivial generalization of the class of discrete spaces. A topological space is said to be extremally disconnected if the closure of every open set is open. The same definition is adapted by Császár [3] in generalized topological spaces as follows: Let $X$ be a set and $\mathcal{P}(X)$ be the power set of $X$. A subset $\mu$ of $\mathcal{P}(X)$ is called generalized topology (GT) on $X$ if $\mu$ is closed under arbitrary unions and, in that case, $(X, \mu)$ is called a generalized topological space (GTS). The elements of $\mu$ are called $\mu$-open sets and their complements are called $\mu$-closed sets. The closure of a set $A$, denoted by $c_{\mu} A$, is the intersection of $\mu$-closed sets containing $A$. A GTS $(X, \mu)$ is called extremally $\mu$-disconnected if $c_{\mu} U \in \mu$ for each $\mu$-open set $U$. Our main argument on which this entire paper is based is that $c_{\mu} U$, for any $U \in \mu$, is never in $\mu$ unless $X \in \mu$. Therefore, if $(X, \mu)$ is not strong, that is, $X \notin \mu$, then $(X, \mu)$ is not extremally $\mu$-disconnected since $c_{\mu} \emptyset=X-M_{\mu}$ where $M_{\mu}=\cup\{U: U \in \mu\}$ is not $\mu$-open. Hence, the notion of extremally $\mu$-disconnectedness does not act as a classification device in the class of non strong generalized topological spaces. This does not seem to be a very satisfactory situation. To rectify the situation, we have modified the above definition as follows: A GTS $(X, \mu)$ is said to be extremally $\mu$-disconnected if $c_{\mu} U \cap M_{\mu} \in \mu$ for each $\mu$-open set $U$. This definition of course reduces to the standard one if $\mu$ is a topology on $X$. The present paper discusses analogues of various properties of extremally $\mu$-disconnected generalized topological spaces.

There is another direction in which this paper achieves further generalizations. In a GTS $(X, \mu)$, a subset $A$ of $X$ is called $\mu$-semi-open if $A \subseteq c_{\mu} i_{\mu} A, \mu$-preopen if

$M S C$ (2010): primary 54A05; secondary 54D15.

Keywords: generalized topological spaces, extremally $\mu$-disconnectedness, $\mu$-connectedness, generalized open sets, $\mu$-upper(lower) semi-continuous mappings.

The second author acknowledges the fellowship grant of University Grant Commission, India. 
$A \subseteq i_{\mu} c_{\mu} A, \mu$ - $\alpha$-open if $A \subseteq i_{\mu} c_{\mu} i_{\mu} A$, and $\mu$ - $\lambda$-open if $A \subseteq c_{\mu} i_{\mu} c_{\mu} A$ [1] : here, $i_{\mu} A$, the interior of $A$, is the union of all $\mu$-open sets contained in $A$. In a topological space, the union of open sets, semi-open sets, preopen sets, $\alpha$-open sets, and $\lambda$ open sets is the same set, which is equal to $X$. If a $\operatorname{GTS}(X, \mu)$ is not strong, the union of $\mu$-open sets is $M_{\mu} \neq X$ whereas, since $X$ is $\mu$-semi-open, the union of $\mu$-semi-open sets is $X$. The situation for the class of $\mu$ - $\lambda$-open sets will be similar. Consequently, we modify the definition of a $\mu$-semi-open set $A$ as follows: $A \subseteq c_{\mu} i_{\mu} A \cap M_{\mu}[8]$ and that of a $\mu$ - $\lambda$-open set as follows : $A \subseteq c_{\mu} i_{\mu} c_{\mu} A \cap M_{\mu}$. Note that, if a GT space is strong, the newly defined notions coincide with the corresponding notion defined above. In this more general framework, we have obtained every result in Sharma [5] and related results in Császár [1,3].

There is a third direction in which further generalization is achieved. Sharma [5] has defined generalized upper semi-continuous(lower semi-continuous) function from a $\operatorname{GTS}(X, \mu)$ to the real line $\mathbb{R}$ and gave an example showing that these two notions together cannot be equivalent to the notion of generalized continuity [2]. We have replaced the real line $\mathbb{R}$ with a generalized topological space $(R, \tau[a, b])$, where $R$ denotes the set of real numbers, $a, b \in R$ and $\tau[a, b]$ is the GT generated by the generalized basis $\mathcal{B}$ that consist of left open rays $[a, c)$ and right open rays $(c, b], a<c<b$. This GT is appropriate to obtain the above equivalence. This also ensures that, in an extremally $\mu$-disconnected GTS $(X, \mu)$, there is a rich supply of $\mu$-upper(lower) semi-continuous functions.

The paper is organized as follows. Section 2 contains basic notions and notation used in the paper. In Section 3, we obtain equivalences shown in [5]. Section 4 deals with the $\mu$-upper( $\mu$-lower) semi-continuous functions. Section 5 provides examples of extremally $\mu$-disconnected generalized topological spaces.

\section{Preliminaries}

Let $X$ be a set. A subset $\mathcal{B}$ of $\mathcal{P}(X)$ is called a generalized basis for $X$ [4]. The collection $\mu$ of all unions of elements of $\mathcal{B}$ is a GT on $X$ called the generalized topology generated by $\mathcal{B}$. $(X, \mu)$ shall be used generically to denote a generalized topological space.

Lemma 2.1. Let $(X, \mu)$ be a GT-space and $A, B \subseteq X$. Then, the following statements hold.

(i) $x \in c_{\mu} A$ if and only if $x \in U \in \mu$ implies $U \cap A \neq \emptyset$.

(ii) If $U, V \in \mu$ and $U \cap V=\emptyset$, then $c_{\mu} U \cap V=\emptyset$ and $U \cap c_{\mu} V=\emptyset$.

(iii) $c_{\mu} A=X-i_{\mu}(X-A)$ for any $A \subseteq X$.

(iv) $c_{\mu} A=c_{\mu}\left(A \cap M_{\mu}\right)$.

(v) For any set $A \subseteq X, i_{\mu} c_{\mu} i_{\mu} c_{\mu} A=i_{\mu} c_{\mu} A$ and $c_{\mu} i_{\mu} c_{\mu} i_{\mu} A=c_{\mu} i_{\mu} A$.

Recall that a set $A$ is said to be $\mu$-semi-open if $A \subseteq c_{\mu} i_{\mu} A \cap M_{\mu}$, $\mu$-preopen if $A \subseteq i_{\mu} c_{\mu} A, \mu$ - $\alpha$-open if $A \subseteq i_{\mu} c_{\mu} i_{\mu} A$ and $\mu$ - $\beta$-open if $A \subseteq c_{\mu} i_{\mu} c_{\mu} A \cap M_{\mu}$. The collection of all $\mu$-semi-open ( $\mu$-preopen, $\mu$ - $\alpha$-open, $\mu$ - $\beta$-open) sets are denoted by $s_{\mu},\left(\pi_{\mu}, \alpha_{\mu}, \beta_{\mu}\right)$. These sets are GT's on $X$ and the following inclusions hold.

Theorem 2.2.

(ii) $\alpha_{\mu} \subseteq \pi_{\mu} \subseteq \beta_{\mu}$.

(i) $\mu \subseteq \alpha_{\mu} \subseteq s_{\mu} \subseteq \beta_{\mu}$. 
The sets $U$ and $V$ in a GT space $(X, \mu)$ are said to be $\mu$-separated if $c_{\mu} U \cap V=\emptyset$ and $U \cap c_{\mu} V=\emptyset$.

A subset $S$ in a GT-space $(X, \mu)$ is said to be $\mu$-connected [7] if $S \cap M_{\mu}=U \cup V$ where $U$ and $V$ are $\mu$-separated sets implies $U=\emptyset$ or $V=\emptyset .(X, \mu)$ is said to be $\mu$-connected if it is a $\mu$-connected subset of itself.

The following lemmas are immediate.

Lemma 2.3. If $\mu$ and $\mu^{\prime}$ are GTs on a set $X$, then $\mu \subseteq \mu^{\prime}$ implies $c_{\mu^{\prime}} A \subseteq c_{\mu} A$ for all $A \subseteq X$.

Lemma 2.4. Let $\mu$ and $\mu^{\prime}$ be GTs on a set $X$ and $\mu \subseteq \mu^{\prime}$. If $U$ and $V$ are $\mu$-separated, then $U$ and $V$ are $\mu^{\prime}$-separated.

Theorem 2.5. Let $\mu$ and $\mu^{\prime}$ be GTs on a set $X$ with $\mu \subseteq \mu^{\prime}$. Then, a $\mu^{\prime}-$ connected set is $\mu$-connected.

Theorem 2.6 ([7]). The following statements are equivalent.

(i) $(X, \mu)$ is $\mu$-connected.

(ii) If $M_{\mu}=G \cup G^{\prime}, G, G^{\prime} \in \mu, G \cap G^{\prime}=\emptyset$, then $G=\emptyset$ or $G^{\prime}=\emptyset$.

A GT-space $(X, \mu)$ is called $\mu$-irreducible [5] if, for each non-empty pair of $\mu$ open sets $U$ and $V, U \cap V \neq \emptyset$.

In view of 2.5 , the following implications are immediate.

$$
\begin{array}{ccc}
\beta_{\mu} \text {-connectedness } & \Rightarrow & \pi_{\mu} \text {-connectedness } \\
\Downarrow & & \Downarrow \\
s_{\mu} \text {-connectedness } & \Rightarrow & \mu \text {-connectedness } \\
& & \mathbb{1} \\
& & \alpha_{\mu} \text {-connectedness }
\end{array}
$$

Theorem 2.7. $\pi_{\beta_{\mu}}=\beta_{\beta_{\mu}}=\beta_{\mu}$.

Proof. Since $A \subseteq c_{\mu} A, c_{\beta_{\mu}} A \subseteq c_{\beta_{\mu}} c_{\mu} A \subseteq c_{\mu} c_{\mu} A=c_{\mu} A$ by Lemma 2.3. If $B \in \beta_{\mu}$ and $B \subseteq c_{\beta_{\mu}} A$, then $B \subseteq c_{\mu} i_{\mu} c_{\mu} B \cap M_{\mu}$ and $B \subseteq c_{\mu} A$. So that $B \subseteq$ $c_{\mu} i_{\mu} c_{\mu} c_{\mu} A \cap M_{\mu}=c_{\mu} i_{\mu} c_{\mu} A \cap M_{\mu}$. Hence, $i_{\beta_{\mu}} c_{\beta_{\mu}} A \subseteq c_{\mu} i_{\mu} c_{\mu} A \cap M_{\mu}$. On the other hand $X-c_{\mu} i_{\mu} c_{\mu} A \subseteq X-i_{\mu} c_{\mu} i_{\mu} c_{\mu} A=c_{\mu}\left(X-c_{\mu} i_{\mu} c_{\mu} A\right)=c_{\mu} i_{\mu}\left(X-i_{\mu} c_{\mu} A\right)=$ $c_{\mu} i_{\mu}\left(X-i_{\mu} c_{\mu} i_{\mu} c_{\mu} A\right)=c_{\mu} i_{\mu} c_{\mu}\left(X-c_{\mu} i_{\mu} c_{\mu} A\right)$. Since $X-c_{\mu} i_{\mu} c_{\mu} A \subseteq M_{\mu}, X-$ $c_{\mu} i_{\mu} c_{\mu} A \in \beta_{\mu}$. This together with $i_{\beta_{\mu}} c_{\beta_{\mu}} A \subseteq c_{\mu} i_{\mu} c_{\mu} A \cap M_{\mu}$ gives the inclusion $c_{\beta_{\mu}} i_{\beta_{\mu}} c_{\beta_{\mu}} A \subseteq c_{\beta_{\mu}}\left(c_{\mu} i_{\mu} c_{\mu} A \cap M_{\mu}\right) \subseteq c_{\beta_{\mu}}\left(c_{\mu} i_{\mu} c_{\mu} A\right)=c_{\mu} i_{\mu} c_{\mu} A$, that is, $\beta_{\beta_{\mu}} \subseteq \beta_{\mu}$. Also $\beta_{\mu} \subseteq \pi_{\beta_{\mu}} \subseteq \beta_{\beta_{\mu}} \subseteq \beta_{\mu}$ by 2.2 .

Corollary 2.8. $\alpha_{\beta_{\mu}}=s_{\beta_{\mu}}=\beta_{\mu}$.

Proof. $\beta_{\mu} \subseteq \alpha_{\beta_{\mu}} \subseteq s_{\beta_{\mu}} \subseteq \beta_{\beta_{\mu}}=\beta_{\mu}$ by 2.2 and 2.7 .

\section{Extremally $\mu$ - DisconneCted Generalized topological SPACES}

Definition 3.1. A GTS $(X, \mu)$ is called extremally $\mu$-disconnected if $c_{\mu} U \cap$ $M_{\mu} \in \mu$ for every $U \in \mu$.

Theorem 3.2. A GTS $(X, \mu)$ is extremally $\mu$-disconnected if and only if, for any disjoint $\mu$-open sets $U$ and $V, c_{\mu} U \cap c_{\mu} V \cap M_{\mu}=\emptyset$. 
Proof. Let $(X, \mu)$ be extremally $\mu$-disconnected and $U$ and $V$ be disjoint $\mu$ open sets. Thus, $c_{\mu} U \cap V=\emptyset$ and $U \cap c_{\mu} V=\emptyset$. Then, $c_{\mu} U \cap M_{\mu} \cap V=\emptyset$ and $U \cap c_{\mu} V \cap M_{\mu}=\emptyset$. Since $c_{\mu} U \cap M_{\mu} \in \mu$, it follows that $c_{\mu} U \cap c_{\mu} V \cap M_{\mu}=\emptyset$.

Conversely, assume that $c_{\mu} U \cap c_{\mu} V \cap M_{\mu}=\emptyset$. Let $W \in \mu$. If $c_{\mu} W=X-M_{\mu}$ then $c_{\mu} W \cap M_{\mu}=\emptyset \in \mu$. Now $W$ and $X-c_{\mu} W$ are disjoint so that $c_{\mu} W \cap$ $c_{\mu}\left(X-c_{\mu} W\right) \cap M_{\mu}=\emptyset$. Hence, $c_{\mu} W \cap M_{\mu} \subseteq X-c_{\mu}\left(X-c_{\mu} W\right)=i_{\mu} c_{\mu} W$. Since $i_{\mu} c_{\mu} W \subseteq c_{\mu} W \cap M_{\mu}, c_{\mu} W \cap M_{\mu} \in \mu$.

Theorem 3.3. A GTS $(X, \mu)$ is extremally $\mu$-disconnected if and only if, for each $U \in \mu$ and $\mu$-closed set $F$ such that $U \subseteq F$, there exist $a V_{1} \in \mu$ and $a$ $\mu$-closed set $F_{1}$ such that $U \subseteq F_{1} \cap M_{\mu} \subseteq V_{1} \subseteq F$.

Proof. Let $(X, \mu)$ be extremally $\mu$-disconnected. Let $U \in \mu$ and $F$ be a $\mu$-closed set with $U \subseteq F$. Then, $U \cap(X-F)=\emptyset$ and, by $3.2 c_{\mu} U \cap c_{\mu}(X-F) \cap M_{\mu}=$ $\emptyset$, that is, $c_{\mu} U \cap M_{\mu} \subseteq X-c_{\mu}(X-F)$. Since $i_{\mu} F=X-c_{\mu}(X-F) \subseteq F$, $U \subseteq c_{\mu} U \cap M_{\mu} \subseteq i_{\mu} F \subseteq F$. Conversely, let $U$ and $V$ be disjoint $\mu$-open sets. Then, $U \subseteq X-V$. Then, by our assumption, there exist a $V_{1} \in \mu$ and a $\mu$ closed set $F$ such that $U \subseteq F_{1} \cap M_{\mu} \subseteq V_{1} \subseteq(X-V)$. Then, it follows that $c_{\mu} U \cap c_{\mu} V \cap M_{\mu}=\emptyset$.

Theorem 3.4. If $(X, \mu)$ is an extremally $\mu$-disconnected GTS, then the following statements are equivalent:

(i) $\left(X, \pi_{\mu}\right)$ is $\pi_{\mu}$-connected.

(ii) $\left(X, \beta_{\mu}\right)$ is $\beta_{\mu}$-connected.

(iii) $\left(X, s_{\beta_{\mu}}\right)$ is $s_{\beta_{\mu}}$-connected.

(iv) $\left(X, \beta_{\mu}\right)$ is $\beta_{\mu}$-irreducible.

Proof. (i) $\Rightarrow$ (ii) Suppose that $\left(X, \beta_{\mu}\right)$ is not $\beta_{\mu}$-connected. Then, by 2.6 , there are disjoint non-empty $\beta_{\mu}$-open sets $U$ and $V$ such that $M_{\beta_{\mu}}=U \cup V$. Then, $U \subseteq$ $c_{\mu} i_{\mu} c_{\mu} U \cap M_{\mu}$ and $V \subseteq c_{\mu} i_{\mu} c_{\mu} V \cap M_{\mu}$. Since $(X, \mu)$ is extremally $\mu$-disconnected, $i_{\mu}\left(c_{\mu} i_{\mu} c_{\mu} U \cap M_{\mu}\right)=c_{\mu} i_{\mu} c_{\mu} U \cap M_{\mu}$ and $i_{\mu}\left(c_{\mu} i_{\mu} c_{\mu} V \cap M_{\mu}\right)=c_{\mu} i_{\mu} c_{\mu} V \cap M_{\mu}$. Therefore, $U \subseteq i_{\mu} c_{\mu} i_{\mu} c_{\mu} U=i_{\mu} c_{\mu} U$. Similarly, $V \subseteq i_{\mu} c_{\mu} U$. So $U$ and $V$ are $\pi_{\mu^{-}}$open, which means that $\left(X, \pi_{\mu}\right)$ is not $\pi_{\mu}$-connected.

(ii) $\Leftrightarrow$ (iii) holds because $s_{\beta_{\mu}}=\beta_{\mu}$ by 2.8 .

(ii) $\Rightarrow$ (iv) Suppose that $\left(X, \beta_{\mu}\right)$ is not $\beta_{\mu}$-irreducible. Then, there are non-empty disjoint $\beta_{\mu}$-open sets $U$ and $V$. Let $P=c_{\mu} U \cap M_{\mu}$ and $Q=X-c_{\mu} U$. Since $U \subseteq$ $c_{\mu} i_{\mu} c_{\mu} U \cap M_{\mu}, c_{\mu} U \subseteq c_{\mu}\left(c_{\mu} i_{\mu} c_{\mu} U \cap M_{\mu}\right)=c_{\mu} c_{\mu} i_{\mu} c_{\mu} U=c_{\mu} i_{\mu} c_{\mu} U=c_{\mu} i_{\mu} c_{\mu}\left(c_{\mu} U\right)=$ $c_{\mu} i_{\mu} c_{\mu}\left(c_{\mu} U \cap M_{\mu}\right)$ by 2.1. Therefore, $c_{\mu} U \cap M_{\mu} \subseteq c_{\mu} i_{\mu} c_{\mu}\left(c_{\mu} U \cap M_{\mu}\right) \cap M_{\mu}$. Consequently, $P \in \beta_{\mu}$. Since $\mu \subseteq \beta_{\mu}, Q \in \beta_{\mu}$. Since $M_{\mu}=M_{\beta_{\mu}}, M_{\beta_{\mu}}=P \cup Q$. So, $\left(X, \beta_{\mu}\right)$ is not $\beta_{\mu}$-connected.

(iv) $\Rightarrow$ (i) holds because $\pi_{\mu} \subseteq \beta_{\mu}$ by 2.2 .

Theorem 3.5. In an extremally $\mu$-disconnected $\operatorname{GTS}(X, \mu)$, the following statements hold:

(i) $\beta_{\mu}=\pi_{\mu}$.

(ii) $s_{\mu}=\alpha_{\mu}$.

(iii) $s_{\mu} \subseteq \pi_{\mu}$. 
Proof. (i) $\pi_{\mu} \subseteq \beta_{\mu}$ by 2.2. Let $A \in \beta_{\mu}$. Then, $A \subseteq c_{\mu} i_{\mu} c_{\mu} A \cap M_{\mu}$. Since $X$ is extremally $\mu$-disconnected, $c_{\mu} i_{\mu} c_{\mu} A \cap M_{\mu} \in \mu$. Therefore, $i_{\mu}\left(c_{\mu} i_{\mu} c_{\mu} A \cap M_{\mu}\right)=$ $c_{\mu} i_{\mu} c_{\mu} A \cap M_{\mu}$. Thus, $A \subseteq i_{\mu} c_{\mu} i_{\mu} c_{\mu} A=i_{\mu} c_{\mu} A$.

(ii) $\alpha_{\mu} \subseteq s_{\mu}$ by 2.2. So, we will prove that $s_{\mu} \subseteq \alpha_{\mu}$. $A \in s_{\mu}$ implies $A \subseteq$ $c_{\mu} i_{\mu} A \cap M_{\mu} \in \mu$. Therefore, $A \subseteq i_{\mu}\left(c_{\mu} i_{\mu} A \cap M_{\mu}\right) \subseteq i_{\mu} c_{\mu} i_{\mu} A$.

(iii) holds by (i) and 2.2.

Corollary 3.6. For an extremally $\mu$-disconnected $\operatorname{GTS}(X, \mu)$, the following three conditions are equivalent:

(i) $\left(X, \pi_{\mu}\right)$ is irreducible,

(ii) $\left(X, \beta_{\mu}\right)$ is $\beta_{\mu}$-irreducible,

(iii) $\left(X, \beta_{\mu}\right)$ is $\beta_{\mu}$-connected.

A point $x \in X$ of a $\operatorname{GTS}(X, \mu)$ is a point of extremal $\mu$-disconnectedness of $X$ if there are no disjoint $\mu$-open sets $U$ and $V$ such that $x \in c_{\mu} U \cap c_{\mu} V \cap M_{\mu}$. Observe that every point in $X-M_{\mu}$ is a point of extremal $\mu$-disconnectedness.

Theorem 3.7. A GTS $(X, \mu)$ is extremally $\mu$-disconnected space if and only if every point of $X$ is a point of extremal $\mu$-disconnectedness.

Theorem 3.8. An extremally $\mu$-disconnected $G T S(X, \mu)$ is $\mu$-connected if and only if $M, N \in \mu, M \neq \emptyset, N \neq \emptyset$ imply $M \cap N \neq \emptyset$.

Proof. The condition is clearly sufficient in view of 2.6 and the property of extremal $\mu$-disconnectedness is not required here. Conversely, we assume that $U, V \in$ $\mu, U \neq \emptyset, V \neq \emptyset$ imply $U \cap V=\emptyset$. Then, $\emptyset \neq U \subseteq c_{\mu} U \cap M_{\mu} \subseteq(X-V) \cap M_{\mu} \neq$ $M_{\mu}$ and $c_{\mu} U \cap M_{\mu} \in \mu$ because $(X, \mu)$ is extremally $\mu$-disconnected GTS. So that $M_{\mu}=\left(c_{\mu} U \cap M_{\mu}\right) \cup\left(X-c_{\mu} U\right)$ which contradicts the $\mu$-connectedness of $(X, \mu)$.

\section{4. $(\mu, \tau)$-UPPER (LOWER) SEMI-CONTINUOUS FUNCTIONS}

Definition 4.1. Let $(X, \mu)$ be a GTS and $R$ be the set of real numbers with GT $\tau[a, b], a, b \in R$, generated by the generalized basis $\mathcal{B}$ consisting of left open rays $[a, c)$ and right open rays $(c, b], a<c<b$. A function $f:(X, \mu) \rightarrow$ $(R, \tau[a, b])$ is called $(\mu, \tau)$-upper semi-continuous $((\mu, \tau)$-lower semi-continuous $)$ if $f^{-1}([a, c))\left(f^{-1}((c, b])\right.$ is $\mu$-open for every $c$ with $a<c<b$.

Let $(X, \mu)$ and $(Y, \tau)$ be GTSs. A mapping $f: X \rightarrow Y$ is said to be $(\mu, \tau)$ continuous ([2]) if $f^{-1}(U)$ is $\mu$-open for each $\tau$-open set $U \subseteq Y$.

Theorem 4.2. A function $f:(X, \mu) \rightarrow(R, \tau[a, b])$ is $(\mu, \tau)$-continuous if and only if $f$ is both $(\mu, \tau)$-upper semi-continuous and $(\mu, \tau)$-lower semi-continuous.

Proof. Let $G$ be $\tau$-open. Then, $G$ is a union of $\mu$-open sets in $\mathcal{B}$. Since $f$ is both $(\mu, \tau)$-upper semi-continuous and $(\mu, \tau)$-lower semi-continuous, the inverses of these $\mu$-open sets in $\mathcal{B}$ are $\mu$-open. Hence, $f^{-1}(G)$ is $\mu$-open and so $f$ is $(\mu, \tau)$ continuous. The converse is obvious.

Theorem 4.3. A function $f:(X, \mu) \rightarrow(R, \tau[a, b])$ is $(\mu, \tau)$-upper semicontinuous if and only if $c f:(X, \mu) \rightarrow(R, \tau[c a, c b])(c f:(X, \mu) \rightarrow(R, \tau[c b, c a]))$ is $(\mu, \tau)$-upper (lower) semi-continuous for every $c>0(c<0)$. 
Theorem 4.4. A function $f:(X, \mu) \rightarrow(R, \tau[a, b])$ is $(\mu, \tau)$ - lower semicontinuous if and only if $c f:(X, \mu) \rightarrow(R, \tau[c a, c b])(c f:(X, \mu) \rightarrow(R, \tau[c b, c a]))$ is $(\mu, \tau)$-lower (upper) semi-continuous for every $c>0(c<0)$.

Theorem 4.5. A function $f:(X, \mu) \rightarrow(R, \tau)[a, b]$ is $(\mu, \tau)$ - upper (lower) semi-continuous if and only if $f+t:(X, \mu) \rightarrow(R, \tau[a+t, b+t])$ is $(\mu, \tau)$-upper (lower) semi-continuous for every $t \in \mathbb{R}$.

In the following theorem, the condition of strong GTS is not required, thereby, improving Theorem 2.5 [5].

Theorem 4.6. Let $(X, \mu)$ be GTS. Then, a subset $A \subseteq X$ is $\mu$-open ( $\mu$-closed) if and only if its characteristic function $\chi_{A}:(X, \mu) \rightarrow(R, \tau[0,1])$ is $(\mu, \tau)$-lower semi-continuous $((\mu, \tau)$-upper semi-continuous).

Theorem 4.7. Let $(X, \mu)$ be an extremally $\mu$-disconnected GTS. Let $U$ and $V$ be two disjoint $\mu$-open sets. Then, there exists a $(\mu, \tau)$-upper semi-continuous function $f:(X, \mu) \rightarrow(R, \tau[0,1])$ such that $f(U)=\{0\}$ and $f(V)=\{1\}$.

Proof. Since $U \subseteq X-V$, by 3.3 , there exist a $G_{1 / 2} \in \mu$ and a $\mu$-closed set $F_{1 / 2}$ such that $U \subseteq F_{1 / 2} \cap M_{\mu} \subseteq G_{1 / 2} \subseteq X-V$

Again, since $U \subseteq F_{1 / 2}$ and $G_{1 / 2} \subseteq X-V$, there exist $\mu$-open sets $G_{1 / 4}, G_{3 / 4}$ and $\mu$-closed sets $F_{1 / 4}, F_{3 / 4}$ such that Thus,

$$
U \subseteq F_{1 / 4} \cap M_{\mu} \subseteq G_{1 / 4} \subseteq F_{1 / 2} \text { and } G_{1 / 2} \subseteq F_{3 / 4} \cap M_{\mu} \subseteq G_{3 / 4} \subseteq X-V
$$

$$
U \subseteq F_{1 / 4} \cap M_{\mu} \subseteq G_{1 / 4} \subseteq F_{1 / 2} \cap M_{\mu} \subseteq G_{1 / 2} \subseteq F_{3 / 4} \cap M_{\mu} \subseteq G_{3 / 4} \subseteq X-V
$$

By induction, for each dyadic rational number of the form $t=\frac{m}{2^{n}}, n=1,2, \ldots$ and $m=1,2, \ldots 2^{n}-1$, we may show that, for $t_{1}<t_{2}$, there are $\mu$-open sets $G_{t_{1}}$ and $G_{t_{2}}$ and $\mu$-closed sets $F_{t_{1}}$ and $F_{t_{2}}$ such that

$$
U \subseteq F_{t_{1}} \cap M_{\mu} \subseteq G_{t_{1}} \subseteq F_{t_{2}} \cap M_{\mu} \subseteq G_{t_{2}} \subseteq X-V
$$

Now we define a function $f:(X, \mu) \rightarrow(R, \tau[0,1])$ as follows:

$$
f(x)= \begin{cases}0, & \text { if } x \in G_{t} \text { for all } t, \\ \sup \left\{t: x \notin G_{t}\right\}, & \text { if } x \in M_{\mu}-\cap_{t} G_{t}, \\ \alpha \in R-[0,1], & \text { if } x \in X-M_{\mu} .\end{cases}
$$

Then, $f(U)=0$ and $f(V)=1$. We will show that $f$ is $(\mu, \tau)$-upper semi-continuous, that is, $f^{-1}([0, a)), 0<a<1$, is $\mu$ - open. Now $x \in f^{-1}([0, a))$ implies $f(x)<a$. So, there must be a dyadic rational $t<a$ such that $x \in G_{t}$. Thus, $f^{-1}([0, a)) \subseteq \cup_{t<a} G_{t}$. On the other hand, if $x \in \cup_{t<a} G_{t}$, then $x \in G_{t_{0}}$ for some $t_{0}<a, f(x) \leq t_{0}<a$. So, $x \in f^{-1}([0, a))$. Therefore, $f$ is $(\mu, \tau)$-upper semi-continuous.

Corollary 4.8. Let $(X, \mu)$ be an extremally $\mu$-disconnected GTS. Let $U$ and $V$ be two disjoint $\mu$-open sets. Then, there exists a $(\mu, \tau)$-lower semi-continuous function $f:(X, \mu) \rightarrow(R, \tau[0,1])$ such that $f(U)=\{0\}$ and $f(V)=\{1\}$.

Proof. By Theorem 4.7, there exists a $(\mu, \tau)$-upper semi-continuous function $f$ such that $f(U)=\{1\}$ and $f(V)=\{0\}$. Thus $-f+1$ is $(\mu, \tau)$-lower semi-continuous and $(-f+1)(U)=\{0\}$ and $(-f+1)(V)=\{1\}$. 


\section{EXAmples of EXtremally $\mu$-DisconneCted Generalized topological SPACES}

For every set $X$, let us denote by $\Gamma_{X}$ the collection of all monotone mappings, i.e., mappings $\gamma: \mathcal{P}(X) \rightarrow \mathcal{P}(X)$ such that $A \subseteq B$ implies $\gamma A \subseteq \gamma B$. A mapping $\gamma: \mathcal{P}(X) \rightarrow \mathcal{P}(X)$ is said to be enlarging if $A \subseteq \gamma A$ for each $A$ in $\mathcal{P}(X)$. If $\gamma \in \Gamma_{X}$, the collection of the sets $A$ satisfying $A \subseteq \gamma A$ constitutes a GT on $X$ denoted by $\lambda_{\gamma}$ in [1]. We denote this GT just by $\gamma$ itself. Any set $A \in \gamma$ is called $\gamma$-open and its complement is called $\gamma$-closed. For $\gamma$ and $\gamma^{\prime}$ in $\Gamma_{X}$, we denote the composition $\gamma \circ \gamma^{\prime}$, which is in $\Gamma_{X}$, by $\gamma \gamma^{\prime}$. Thus, $\gamma \gamma^{\prime}=\left\{A \subseteq X: A \subseteq \gamma \gamma^{\prime} A\right\}$ is a GT on $X$.

For the purpose of the next three results, we denote, for a $\operatorname{GTS}(X, \mu)$, the interior $i_{\mu}$ and the closure $c_{\mu}$ by $i$ and $k$, respectively. Of course, both $i$ and $k$ are elements of $\Gamma_{X}$ and $k i \gamma \in \Gamma_{X}$ is enlarging whenever $\gamma: \mathcal{P}(X) \rightarrow \mathcal{P}(X)$ is enlarging.

Lemma 5.1. If $(X, \mu)$ is a GTS and $\gamma \in \Gamma$ is enlarging, then $\mu \subseteq \nu=k i \gamma$.

Proof. See 2.1 of [3].

Theorem 5.2. For an arbitrary $G T S(X, \mu)$, if $\gamma \in \Gamma$ is enlarging, then $(X, \nu)$ is extremally $\nu$-disconnected GTS for $\nu=k i \gamma$.

Proof. If $A$ is $\nu$-open, then $A \subseteq k i \gamma A$ and the set $k i \gamma A$ is $\nu$-closed by 5.1 . Hence, $c_{\nu} A \cap M_{\mu} \subseteq k i \gamma A \subseteq k i \gamma\left(c_{\nu} A \cap M_{\nu}\right)$ since $A \subseteq c_{\nu} A \cap M_{\nu}$.

Theorem 5.3. For a $\operatorname{GTS}(X, \mu)$

(i) $\left(X, s_{\mu}\right)$ is extremally $s_{\mu}$-disconnected.

(ii) $\left(X, \beta_{\mu}\right)$ is extremally $\beta_{\mu}$-disconnected.

Proof. Apply 5.2 to $\gamma=i d$, the identity mapping and $\gamma=k$, respectively.

A $\operatorname{GTS}(X, \mu)$ is said to be $\mu$-discrete if $x \in M_{\mu}$ implies that $\{x\}$ is $\mu$-open.

Theorem 5.4. If $(X, \mu)$ is $\mu$-discrete, then $(X, \mu)$ is extremally $\mu$-disconnected.

Acknowledgement. The authors are very grateful to the referee for his observations which have improved the value of this paper.

\section{REFERENCES}

[1] Á. Császár, Generalized open sets, Acta Math. Hungar. 125 (2000), 309-335.

[2] Á. Császár, Generalized topology, generalized continuity, Acta Math. Hungar. 96 (2002), 351-357.

[3] Á. Császár, Extremally disconnected generalized topologies, Annales Univ. Sci. Budapest 47 (2004), 91-96.

[4] Á. Császár, Product of generalized topologies, Acta Math. Hungar. 123 (2009), 127-132.

[5] R. D. Sarma, On extremally disconnected generalized topologies, Acta Math. Hungar. 134 (2012), 583-588.

[6] M. H. Stone, Algebraic characterization of special boolean rings, Fund. Math. 19 (1937), 123-302.

[7] B. K. Tyagi, H. V.S. Chauhan and R. Choudhary, On $\gamma$-connected sets, International Journal of Computer Applications 113 (2015), 1-3.

[8] B. K. Tyagi and H.V.S. Chauhan, A remark on semi-open sets in generalized topological spaces, communicated. 
Brij Kishore Tyagi, Department of Mathematics, Atmaram Sanatan Dharma College, University of Delhi, New Delhi-110021, India e-mail: brijkishore.tyagi@gmail.com

Harsh V. S. Chauhan, Department of Mathematics, University of Delhi, New Delhi-110007, India

e-mail: harsh.chauhan111@gmail.com 